\title{
Students' Grade Inquiry Kiosk Using Biometric Fingerprint Authentication With SMS Notification To Parents
}

\author{
Mary Jane R. Laranang ${ }^{1}$, Jenefer P. Bermusa ${ }^{2}$, Edward B. Panganiban ${ }^{3}$, Sandy E. Laranang ${ }^{4}$ \\ ${ }^{1,2}$ AMA Computer College, Santiago City, Isabela, Philippines \\ ${ }^{3}$ Isabela State University, Echague, Isabela, Philippines \\ ${ }^{4}$ Quirino State University, Diffun, Quirino, Philippines \\ E-mail: maryjanelaranang@gmail.com ${ }^{1}$, jenbermusa@gmail.com², ebpanganiban@isu.edu.ph ${ }^{3}$, \\ halflife1104@yahoo.com ${ }^{4}$
}

\begin{abstract}
Technological advancements have helped educators and institutions to be an aid in learning, whichhas been an advantage to big institutions as well as the small ones. These advancements have attracted more and more students to enroll in a particular institution, thus being a competitive advantage. With this modernization, more clients depend on the progress of technology, which convinces them to demand more efficient learning. Due to people's demand and lifestyle change, popularity for the development of the advanced technology employed is excellent. Almost everything used has been changed to better standards. A practical example is a grades viewing system, wherein parents need not worry about their children if they are going in or out of school. Therefore, the proponents came up with the idea of developing a system entitled, "Students' Grade Inquiry Kiosk using Biometric Fingerprint Authentication with SMS Notification to Parents." The primary purpose of the system is to provide an automated viewing of student's grade with real-time SMS notification to parents. The Short Message Service (SMS) performs an essential role in an individual's daily lives. This research has successfully established and produced a reporting system to send notifications straightforwardly to the parent's mobile phone via SMS, thereby helping to ensure the data is delivered to all parents or students concerned. They were efficiently integrating this message system to provide a dependable and useful communication medium. The authors have created a system based on the conceptual framework and system architecture identified after defining the problems of the current issue. The researchers used alpha, beta, and acceptance tests to test the reliability of the device. The machine functions, which act as a test method, have been carried out. During the pilot test, the respondents were given a survey questionnaire about the performance of the system. The majority of the respondents rated the system favorably in terms of its functionality, reliability, and usability. This result confirmed that the system had performed its intended use.
\end{abstract}

Key words: Grades, Kiosk, Fingerprint Biometrics, SMS, Authentication, Parents

\section{INTRODUCTION}

In the academe, grading in education represents the standardized measurement of the achievement in the course of a student. Grades can be assigned in letters such as A, B, C, D, or F, and numbers such as 1, 2, 3, 4, or 5. For certain countries, the grades of all existing grades are combined to produce the grade point average ( GPA) for the marking period. The GPA shall be determined by taking into account the number of grade points obtained by a student over a given time separated by the total number of credits received. The GPA can be used by prospective employers or academic institutions to measure and determine candidates or learners. The grade inquiry system involves methods such as compiling grades of one student, announcement of grades and waiting for the scheduled release of grades. The way of announcing exams' grades is an essential topic in learning. For announcing exams' grades on the web, various methods have been proposed by Shirali-Shahreza [1].

For some parents and students, inquiry of grades sometimes lacks the process of monitoring and giving the details of grades of the student in a particular subject. This issue leads to the risk of wrong results of grades of the students. The authors gathered some facts pertaining to the problems being encountered in one school. The registrar and teachers find out the manual system in giving grades to the students are not always reliable. The problems that occurred are loss of internet connection, late submissions of grades of faculty members, and the students or the teacher is not present to discuss why their grades are not available yet. It also happens that the parents of the students are not informed about the grade performance of their child. It is a significant problem wherein students can manipulate or alter their grades. This situation must not be tolerated, and make sure that parents or guardians of the students are given the correct information about their grades. To improve the current system, the researchers conceptualized a method entitled "Students Grade Viewing Kiosk using Biometric Fingerprint Authentication and SMS Notification" intended for the school campus. This study is a grade inquiry system that can monitor and view grades results by both parents and students. 
The proposed system used a kiosk system integrated with fingerprint authentication and SMS notification to the parents. The unique design of the fingerprint makes it suitable for use in automatic detection systems. The fingerprint is composed of a series of grooves and ridges. Once the fingerprint has been identified, the machine can find the minutia points [2]. Such points are traced, and a line is drawn between each point. It produces a diagram of the way each point relates to the other points. The map is then stored as a data stream called a minutiae template in a database for possible comparison with different fingerprints provided. It is useful to note that no fingerprint images are saved on the device during the whole process, and the fingerprint image cannot be recreated from the minutiae sample [3]. Fingerprint biometrics are widely used in the world today. This technology is used for student attendance information system for exam needs [4].

On the other hand, the short message service (SMS) [5], [6], [7] technology is one of the most stable mobile technologies around. Most of the students carry mobile phones with SMS facilities and can be used for teaching and learning [8]. This SMS was used for school event notification [9], Multiple Choice Quizzes [10][11], Student Information Report System [12], Fingerprint Based Students attendance System [13], Student Information System [14], and Computerizing values and Accessing Values [15]. SMS was also utilized in an information system using student portals to send notifications directly to the parent's mobile phones via SMS and thus help in making sure that the information is delivered to all interested parents or students [16]. Furthermore, the SMS-Based grade inquiry system was established by Codilan [17], wherein it focused on managing student grades, which will be encoded in a database system that can be easily accessed and managed by the user or administrator. Compared with the proposed system, our system can maintain and organize records like student information, subjects, and grades. Furthermore, the system provides the system user with a well-organized record for students while inquiring about their grades.

\section{METHODS AND PROCEDURES}

This study involves a conceptual framework and system architecture that serves as guides in the development of the system. These are only some of the methods and procedures used to come up with an organized system application. These are further explained in the next discussion.

\subsection{CONCEPTUAL FRAMEWORK}

Figure 1 shows the conceptual framework of the system. The admin can log in to the system, and have access to importing student's information, update the records, enrolling the students' fingerprint, as well as backup the database. There will be a notification to the parents or guardians if the students are already enrolled in the school. The notification will inform that their number has been registered in the system. The teachers can also login to the system and have access to the importing of the students' grades. The notification of the grades to the parents/guardians will send in the set date and time of the admin. The students can view their grades by tapping in the biometric scanner, and the database will respond if it is correct or not. Students can also have the option of printing their grades.

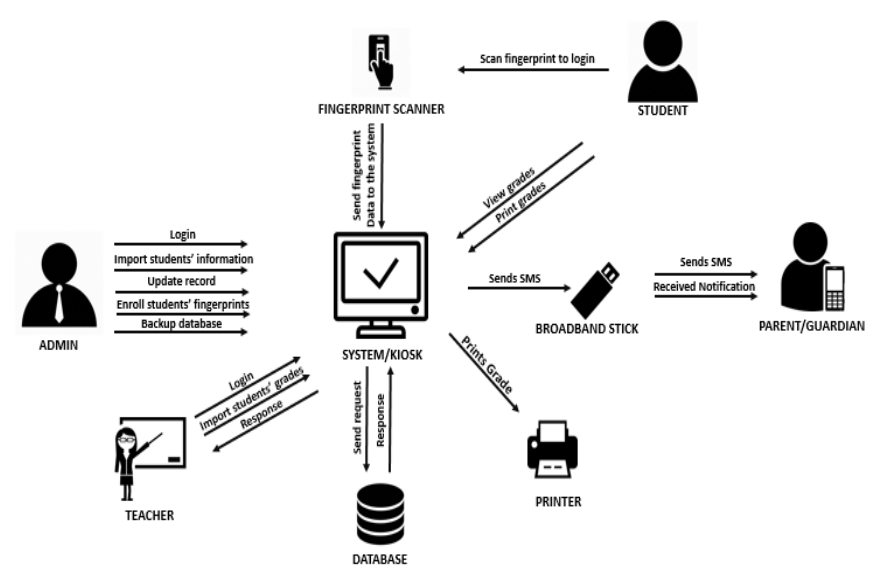

Figure 1: Conceptual Framework

\subsection{SYSTEM ARCHITECTURE}

Figure 2 shows the system architecture of the system. It shows how the system works, from logging-in into the system and encoding the vehicle's plate number as well as printing of the car pass ticket with the corresponding barcode. It also shows the manner of capturing or scanning of car pass ticket and its process flow within the system. The system administrator or the sub-user needs to access the system to encode the car plate number, and the data will be stored in the system's database. The barcode scanner captures the barcode number and sends it to the system [18]. The system verifies the barcode on the system's database. After verification, the system saves in the database the time when the barcode was scanned.

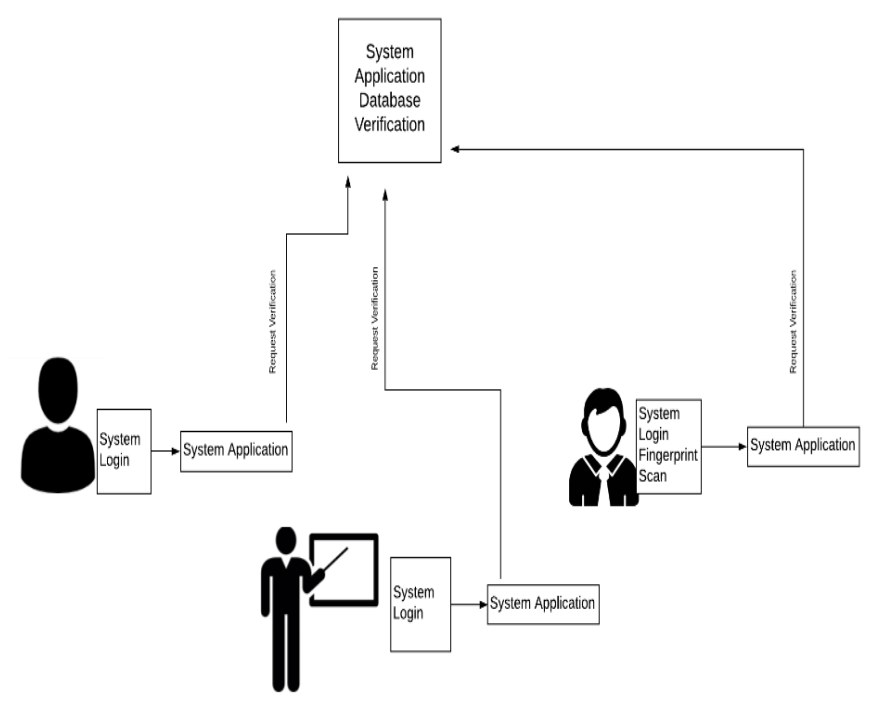

Figure 2: System Architecture 


\section{DISCUSSION OF RESULTS}

\subsection{SOFTWARE DEVELOPMENT}

Figure 3 is the actual main interface of the system administrator. The researchers implemented its pilot testing in AMA Computer College Santiago Campus, particularly to its Kto12 students. The interface displays several functions of the system. These include creating a user that manages all the accounts of the teacher kept in the database. The students' button allows the administrator to manage student records in the database. The grades button allows the administrator to import grades from an excel file format. The backup and restore function will enable the administrator to safeguard the database into other safekeeping. The SMS session allows the administrator to set up a date and schedule for sending grades notification to the parents via SMS. It also includes a password hint button that enables the admin to update their password in case of password recovery. The close app function allows the administrator to exit from the system.

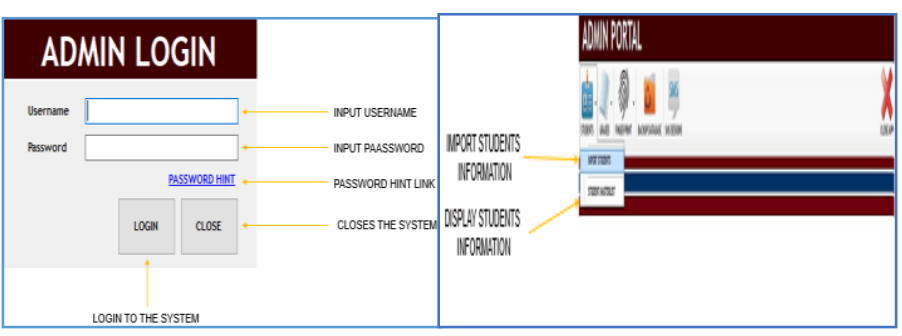

Figure 3: System administrator's interface

Figure 4 is the actual main interface of teachers. The interference display limited only to three functions: the student's button, which allows displaying students list, the grade button to import of grades, and password hint.

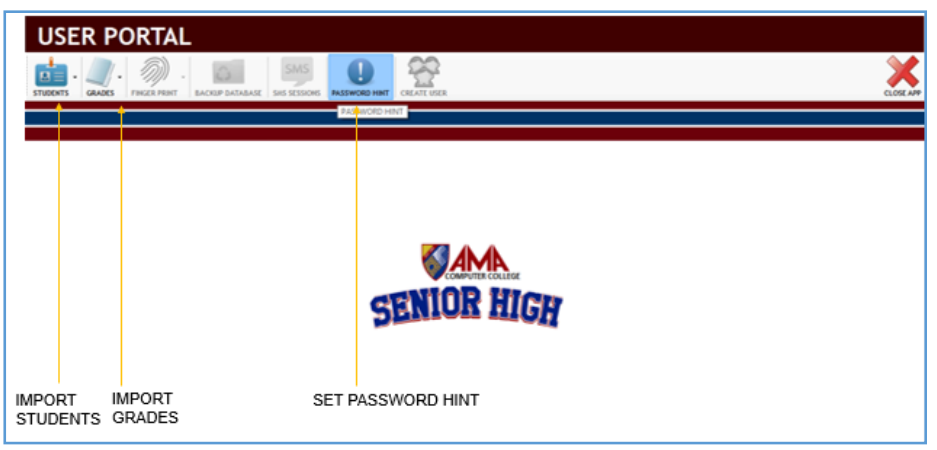

Figure 4: Teacher's main interface

Figure 5 is the actual interface of the student's kiosk. In the given figure, the user interface displays the functions available for the student. The available features are the options to set school year and semester. The print and close buttons are also available.

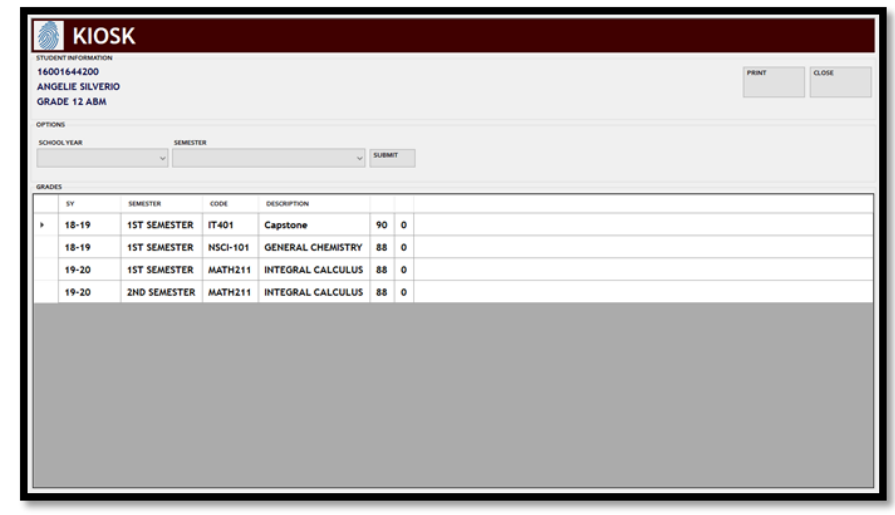

Figure 5: Student's main interface

\subsection{RESULTS OF TESTING}

\subsubsection{ADMINISTRATOR'S AND SUB-USERS ACCOUNTS REGISTRATION TEST}

The account creation tests were conducted for accounts of administrators and sub-users, as illustrated in Table 1. The figure shows an example of 5 administrator's accounts and five sub-users, which were successfully created in the system's database. The ID column pertains to the accounts ID per record, and the NAME is the personal information of the account owner. The USERNAME and PASSWORD columns are the registered security credentials to be used as a login account of users when opening or accessing the system. The LEVEL column determines the role of users per account. The table is a detailed summary of the user's account wherein tests were done to verify its reliability.

\subsubsection{ADMINISTRATOR IMPORTING STUDENT PROFILE TEST}

For importing of student's profile, table 2 shows ten instances of importing records that successfully imported ten records to the system's database. The SID column refers to the id of the student. The STUDENTNAME and YEAR LEVEL AND SECTION columns are the profile of students. The PARENTS/GUARDIAN and PHONE columns are the information of the parents and guardian and their phone number about the student record for grade notification via SMS. The table showed the importing of students had obtained the desired output.

\subsubsection{ADMINISTRATOR ENROLLMENT OF STUDENT'S FINGERPRINT TEST}

For enrollment of student's fingerprint, table 3 shows sample 10 instances were successfully registered in the system's database through the biometric fingerprint scanner. The SID column refers to the id of the student. The STUDENTNAME column indicates the name of the students. The FP column is the captured and recorded fingerprint of the students, which used as authentication access of students in querying their grades in the student's grades inquiry kiosk system. 
Mary Jane R. Laranang et al., International Journal of Emerging Trends in Engineering Research, 8(9), September 2020, 5445 - 5453

Table 1: Account creation test for system user

\begin{tabular}{|c|c|c|c|c|c|}
\hline TRIAL & SID & STUDENTNAME & FP & $\begin{array}{c}\text { EXPECTED } \\
\text { RESULT }\end{array}$ & $\begin{array}{c}\text { ACTUAL } \\
\text { RESULT }\end{array}$ \\
\hline $\mathbf{1}$ & 16001644200 & ANGELIE SILVERIO & BLOB & SUCCESSFUL & SUCCESSFUL \\
\hline $\mathbf{2}$ & 16002497600 & DAISY BRILLANTES & BLOB & SUCCESSFUL & SUCCESSFUL \\
\hline $\mathbf{3}$ & 18002782200 & ELISHA JAMES VAQUILAR & BLOB & SUCCESSFUL & SUCCESSFUL \\
\hline $\mathbf{4}$ & 16002497601 & LOVE CARE & BLOB & SUCCESSFUL & SUCCESSFUL \\
\hline $\mathbf{5}$ & 16004224300 & REJOICE ULANGCA & BLOB & SUCCESSFUL & SUCCESSFUL \\
\hline $\mathbf{6}$ & 18001547635 & MARY JANE LARANANG & BLOB & SUCCESSFUL & SUCCESSFUL \\
\hline $\mathbf{7}$ & 18001325784 & CARLDWIGHT OCUMEN & BLOB & SUCCESSFUL & SUCCESSFUL \\
\hline $\mathbf{8}$ & 18001325785 & BRYAN L. AGUDONG & BLOB & SUCCESSFUL & SUCCESSFUL \\
\hline $\mathbf{9}$ & 18001325786 & MARK T. ANTONIO & BLOB & SUCCESSFUL & SUCCESSFUL \\
\hline $\mathbf{1 0}$ & 18001325787 & JOHN M BALICAO & BLOB & SUCCESSFUL & SUCCESSFUL \\
\hline
\end{tabular}

Table 2: Importing of student's profile to the system's database using excel file format.

\begin{tabular}{|c|c|c|c|c|c|c|c|}
\hline TRIAL & SID & $\begin{array}{c}\text { STUDENTNA } \\
\text { ME }\end{array}$ & $\begin{array}{c}\text { YEAR } \\
\text { LEVEL \& } \\
\text { SECTION }\end{array}$ & $\begin{array}{c}\text { PARENTS } \\
\text { GUARDIANS }\end{array}$ & PHONE & $\begin{array}{c}\text { EXPECTED } \\
\text { RESULT }\end{array}$ & $\begin{array}{l}\text { ACTUAL } \\
\text { RESULT }\end{array}$ \\
\hline 1 & 16001644200 & $\begin{array}{l}\text { ANGELIE } \\
\text { SILVERIO }\end{array}$ & $\begin{array}{c}\text { GRADE } 12 \\
\text { ABM }\end{array}$ & $\begin{array}{c}\text { CELSO APOLTO } \\
\text { SILVERIO }\end{array}$ & 09051721234 & SUCCESSFUL & SUCCESSFUL \\
\hline 2 & 16002497600 & $\begin{array}{c}\text { DAISY } \\
\text { BRILLANTES }\end{array}$ & $\begin{array}{l}\text { GRADE } 12 \\
\text { ABM }\end{array}$ & $\begin{array}{c}\text { ROMMEL } \\
\text { BRILLANTES }\end{array}$ & 09958570948 & SUCCESSFUL & SUCCESSFUL \\
\hline 3 & 18002782200 & $\begin{array}{c}\text { ELISHA } \\
\text { JAMES } \\
\text { VAQUILAR }\end{array}$ & $\begin{array}{l}\text { GRADE } 12 \\
\text { ABM }\end{array}$ & $\begin{array}{l}\text { DOLORES } \\
\text { VAQUILAR }\end{array}$ & 09958570948 & SUCCESSFUL & SUCCESSFUL \\
\hline 4 & 16002497601 & LOVE CARE & $\begin{array}{l}\text { GRADE } 12 \\
\text { ABM }\end{array}$ & $\begin{array}{c}\text { DAISY } \\
\text { BRILLANTES } \\
\end{array}$ & 09399170426 & SUCCESSFUL & SUCCESSFUL \\
\hline 5 & 16004224300 & $\begin{array}{l}\text { REJOICE } \\
\text { ULANGCA }\end{array}$ & $\begin{array}{l}\text { GRADE } 12 \\
\text { ABM }\end{array}$ & ELY ULANGCA & 09667459112 & SUCCESSFUL & SUCCESSFUL \\
\hline 6 & 18001547635 & $\begin{array}{l}\text { MARY JANE } \\
\text { LARANANG }\end{array}$ & $\begin{array}{c}\text { GRADE } 12 \\
\text { ABM }\end{array}$ & $\begin{array}{c}\text { SANDY } \\
\text { LARANANG }\end{array}$ & 09351145975 & SUCCESSFUL & SUCCESSFUL \\
\hline 7 & 18001325784 & $\begin{array}{l}\text { CARLDWIG } \\
\text { HT OCUMEN }\end{array}$ & $\begin{array}{c}\text { GRADE } 12 \\
\text { ABM }\end{array}$ & LYDIA OCUMEN & 09351145975 & SUCCESSFUL & SUCCESSFUL \\
\hline 8 & 18001325785 & $\begin{array}{l}\text { BRYAN L. } \\
\text { AGUDONG }\end{array}$ & $\begin{array}{c}\text { GRADE } 12 \\
\text { ABM }\end{array}$ & $\begin{array}{l}\text { MELLISSA } \\
\text { AGUDONG }\end{array}$ & 09351145975 & SUCCESSFUL & SUCCESSFUL \\
\hline 9 & 18001325786 & $\begin{array}{l}\text { MARK T. } \\
\text { ANTONIO }\end{array}$ & $\begin{array}{c}\text { GRADE } 12 \\
\text { ABM }\end{array}$ & $\begin{array}{c}\text { NESTOR } \\
\text { ANTONIO }\end{array}$ & 09351145975 & SUCCESSFUL & SUCCESSFUL \\
\hline 10 & 18001325787 & $\begin{array}{l}\text { JOHN M } \\
\text { BALICAO }\end{array}$ & $\begin{array}{l}\text { GRADE } 12 \\
\text { ABM }\end{array}$ & $\begin{array}{c}\text { MYRNA } \\
\text { BALICAO }\end{array}$ & 09351145975 & SUCCESSFUL & SUCCESSFUL \\
\hline
\end{tabular}

Table 3: Enrollment of student's fingerprint to the system

\begin{tabular}{|c|c|c|c|c|l|}
\hline TRIAL & SID & STUDENTNAME & FP & $\begin{array}{c}\text { EXPECTED } \\
\text { RESULT }\end{array}$ & $\begin{array}{c}\text { ACTUAL } \\
\text { RESULT }\end{array}$ \\
\hline $\mathbf{1}$ & 16001644200 & ANGELIE SILVERIO & BLOB & SUCCESSFUL & SUCCESSFUL \\
\hline $\mathbf{2}$ & 16002497600 & DAISY BRILLANTES & BLOB & SUCCESSFUL & SUCCESSFUL \\
\hline $\mathbf{3}$ & 18002782200 & ELISHA JAMES VAQUILAR & BLOB & SUCCESSFUL & SUCCESSFUL \\
\hline $\mathbf{4}$ & 16002497601 & LOVE CARE & BLOB & SUCCESSFUL & SUCCESSFUL \\
\hline $\mathbf{5}$ & 16004224300 & REJOICE ULANGCA & BLOB & SUCCESSFUL & SUCCESSFUL \\
\hline $\mathbf{6}$ & 18001547635 & MARY JANE LARANANG & BLOB & SUCCESSFUL & SUCCESSFUL \\
\hline $\mathbf{7}$ & 18001325784 & CARLDWIGHT OCUMEN & BLOB & SUCCESSFUL & SUCCESSFUL \\
\hline $\mathbf{8}$ & 18001325785 & BRYAN L. AGUDONG & BLOB & SUCCESSFUL & SUCCESSFUL \\
\hline $\mathbf{9}$ & 18001325786 & MARK T. ANTONIO & BLOB & SUCCESSFUL & SUCCESSFUL \\
\hline $\mathbf{1 0}$ & 18001325787 & JOHN M BALICAO & BLOB & SUCCESSFUL & SUCCESSFUL \\
\hline
\end{tabular}




\subsubsection{TEACHER IMPORTING GRADES TEST}

For importing of grades, table 4 shows sample 10 instances were successfully imported to the database. The TRIAL column refers to the number of samples conducted. The TEACHER column indicates to the teacher who attended the trial. The SY, CODE, DESCRIPTION YR_SEC, SID, NAME, GRADE, and SEM columns is about the data designated to the account of the students' record. Based on the table, actual results showed that the 431 system works appropriately based on expected outputs.

\subsubsection{STUDENT GRADE INQUIRY TEST}

For grade inquiry, table 5 shows sample 10 instances were successfully log in and displayed queried data with the use of a biometric fingerprint scanner for authenticating student's fingerprint login. The table shows the ten accounts that tested the system with the expected and actual results. On successful login, the system will show the student id, name, year and strand, subjects, and corresponding grades that match the enrolled fingerprint of the student in the database.

\subsubsection{ADMINISTRATOR'S GRADES NOTIFICATION VIA SMS EVALUATION}

For notification of student's grade to the guardians via SMS, table 6 shows ten instances which successfully sends grade of students to the guardians. The SID column refers to the id of the student. The STUDENTNAME and YEAR LEVEL AND SECTION columns are the profile of students. The PARENTS/GUARDIAN and PHONE columns are the information of the parents and guardian and their phone

Table 4: Teacher importing of student's grades to the system's database

\begin{tabular}{|c|c|c|c|c|c|c|c|c|c|c|c|}
\hline $\begin{array}{l}\mathbf{T} \\
\mathbf{R} \\
\mathbf{I} \\
\mathbf{A} \\
\mathbf{L} \\
\end{array}$ & TEACHER & SY & $\begin{array}{l}\text { CO } \\
\text { DE }\end{array}$ & $\begin{array}{l}\text { DESCRIP } \\
\text { TION }\end{array}$ & YR_SEC & SID & NAME & $\begin{array}{c}\text { GR } \\
\text { AD } \\
\text { E }\end{array}$ & SEM & $\begin{array}{c}\text { EXPE } \\
\text { CTED } \\
\text { RESU } \\
\text { LT }\end{array}$ & $\begin{array}{l}\text { ACTUAL } \\
\text { RESULT }\end{array}$ \\
\hline 1 & $\begin{array}{c}\text { SHERYL } \\
\text { RASPADO }\end{array}$ & $\begin{array}{c}18 \\
-1 \\
9\end{array}$ & $\begin{array}{c}\text { EN1 } \\
01\end{array}$ & $\begin{array}{c}\text { BASIC } \\
\text { ENGLISH }\end{array}$ & $\begin{array}{l}\text { GRADE } \\
12 \mathrm{ABM}\end{array}$ & $\begin{array}{c}160016 \\
44200\end{array}$ & $\begin{array}{l}\text { ANGELIE } \\
\text { SILVERIO }\end{array}$ & 88 & $\begin{array}{l}\text { 2ND } \\
\text { SEM }\end{array}$ & $\begin{array}{l}\text { SUCCE } \\
\text { SSFUL }\end{array}$ & $\begin{array}{c}\text { SUCCESSF } \\
\text { UL }\end{array}$ \\
\hline 2 & $\begin{array}{l}\text { SHERYL } \\
\text { RASPADO }\end{array}$ & $\begin{array}{c}18 \\
-1 \\
9 \\
\end{array}$ & $\begin{array}{c}\text { EN1 } \\
01\end{array}$ & $\begin{array}{c}\text { BASIC } \\
\text { ENGLISH }\end{array}$ & $\begin{array}{l}\text { GRADE } \\
12 \text { ABM }\end{array}$ & $\begin{array}{c}180027 \\
82200\end{array}$ & $\begin{array}{c}\text { ELISHA } \\
\text { JAMES } \\
\text { VAQUILAR }\end{array}$ & 98 & $\begin{array}{l}\text { 2ND } \\
\text { SEM }\end{array}$ & $\begin{array}{l}\text { SUCCE } \\
\text { SSFUL }\end{array}$ & $\begin{array}{c}\text { SUCCESSF } \\
\text { UL }\end{array}$ \\
\hline 3 & $\begin{array}{c}\text { SANDY } \\
\text { LARANANG }\end{array}$ & $\begin{array}{c}18 \\
-1 \\
9\end{array}$ & $\begin{array}{c}\text { FIL1 } \\
02\end{array}$ & $\begin{array}{c}\text { FILIPINO } \\
2\end{array}$ & $\begin{array}{l}\text { GRADE } \\
12 \text { ABM }\end{array}$ & $\begin{array}{l}160016 \\
44200\end{array}$ & $\begin{array}{l}\text { ANGELIE } \\
\text { SILVERIO }\end{array}$ & 88 & $\begin{array}{l}\text { 2ND } \\
\text { SEM }\end{array}$ & $\begin{array}{l}\text { SUCCE } \\
\text { SSFUL }\end{array}$ & $\begin{array}{c}\text { SUCCESSF } \\
\text { UL }\end{array}$ \\
\hline 4 & $\begin{array}{c}\text { SANDY } \\
\text { LARANANG }\end{array}$ & $\begin{array}{c}1 \\
8- \\
1 \\
9\end{array}$ & $\begin{array}{c}\text { FIL1 } \\
02\end{array}$ & $\begin{array}{c}\text { FILIPINO } \\
2\end{array}$ & $\begin{array}{l}\text { GRADE } \\
12 \mathrm{ABM}\end{array}$ & $\begin{array}{c}180027 \\
82200\end{array}$ & $\begin{array}{c}\text { ELISHA } \\
\text { JAMES } \\
\text { VAQUILAR }\end{array}$ & 98 & $\begin{array}{l}\text { 2ND } \\
\text { SEM }\end{array}$ & $\begin{array}{l}\text { SUCCE } \\
\text { SSFUL }\end{array}$ & $\begin{array}{c}\text { SUCCESSF } \\
\text { UL }\end{array}$ \\
\hline 5 & $\begin{array}{l}\text { JUNEMA } \\
\text { SAMILING }\end{array}$ & $\begin{array}{c}1 \\
8- \\
1 \\
9 \\
\end{array}$ & $\begin{array}{l}\text { NSC } \\
\text { I-101 }\end{array}$ & $\begin{array}{c}\text { GENERA } \\
\text { L } \\
\text { CHEMIST } \\
\text { RY } \\
\end{array}$ & $\begin{array}{l}\text { GRADE } \\
12 \text { ABM }\end{array}$ & $\begin{array}{c}160016 \\
44200\end{array}$ & $\begin{array}{l}\text { ANGELIE } \\
\text { SILVERIO }\end{array}$ & 88 & $\begin{array}{l}\text { 2ND } \\
\text { SEM }\end{array}$ & $\begin{array}{l}\text { SUCCE } \\
\text { SSFUL }\end{array}$ & $\begin{array}{c}\text { SUCCESSF } \\
\text { UL }\end{array}$ \\
\hline 6 & $\begin{array}{l}\text { JUNEMA } \\
\text { SAMILING }\end{array}$ & $\begin{array}{c}18 \\
-1 \\
9\end{array}$ & $\begin{array}{l}\text { NSC } \\
\text { I-101 }\end{array}$ & $\begin{array}{c}\text { GENERA } \\
\text { L } \\
\text { CHEMIST } \\
\text { RY } \\
\end{array}$ & $\begin{array}{l}\text { GRADE } \\
12 \mathrm{ABM}\end{array}$ & $\begin{array}{c}180027 \\
82200\end{array}$ & $\begin{array}{c}\text { ELISHA } \\
\text { JAMES } \\
\text { VAQUILAR }\end{array}$ & 98 & $\begin{array}{l}\text { 2ND } \\
\text { SEM }\end{array}$ & $\begin{array}{l}\text { SUCCE } \\
\text { SSFUL }\end{array}$ & $\begin{array}{c}\text { SUCCESSF } \\
\text { UL }\end{array}$ \\
\hline 7 & $\begin{array}{c}\text { KARL } \\
\text { NOLASCO }\end{array}$ & $\begin{array}{l}1 \\
8- \\
1 \\
9 \\
\end{array}$ & $\begin{array}{c}\text { MA } \\
\text { TH2 } \\
11\end{array}$ & $\begin{array}{c}\text { INTEGRA } \\
\text { L } \\
\text { CALCUL } \\
\text { US } \\
\end{array}$ & $\begin{array}{l}\text { GRADE } \\
12 \mathrm{ABM}\end{array}$ & $\begin{array}{c}160016 \\
44200\end{array}$ & $\begin{array}{l}\text { ANGELIE } \\
\text { SILVERIO }\end{array}$ & 88 & $\begin{array}{l}\text { 2ND } \\
\text { SEM }\end{array}$ & $\begin{array}{l}\text { SUCCE } \\
\text { SSFUL }\end{array}$ & $\begin{array}{c}\text { SUCCESSF } \\
\text { UL }\end{array}$ \\
\hline 8 & $\begin{array}{c}\text { KARL } \\
\text { NOLASCO }\end{array}$ & $\begin{array}{c}1 \\
8- \\
1 \\
9 \\
\end{array}$ & $\begin{array}{c}\text { MA } \\
\text { TH2 } \\
11\end{array}$ & $\begin{array}{c}\text { INTEGRA } \\
\text { L } \\
\text { CALCUL } \\
\text { US } \\
\end{array}$ & $\begin{array}{l}\text { GRADE } \\
12 \mathrm{ABM}\end{array}$ & $\begin{array}{c}180027 \\
82200\end{array}$ & $\begin{array}{c}\text { ELISHA } \\
\text { JAMES } \\
\text { VAQUILAR }\end{array}$ & 98 & $\begin{array}{l}\text { 2ND } \\
\text { SEM }\end{array}$ & $\begin{array}{l}\text { SUCCE } \\
\text { SSFUL }\end{array}$ & $\begin{array}{c}\text { SUCCESSF } \\
\text { UL }\end{array}$ \\
\hline 9 & $\begin{array}{c}\text { ADONIS } \\
\text { NICOLAS }\end{array}$ & $\begin{array}{c}18 \\
-1 \\
9 \\
\end{array}$ & $\begin{array}{c}\text { EN1 } \\
02\end{array}$ & $\begin{array}{l}\text { WRITE } \\
\text { ENGLISH }\end{array}$ & $\begin{array}{l}\text { GRADE } \\
12 \mathrm{ABM}\end{array}$ & $\begin{array}{l}160016 \\
44200\end{array}$ & $\begin{array}{l}\text { ANGELIE } \\
\text { SILVERIO }\end{array}$ & 88 & $\begin{array}{l}\text { 2ND } \\
\text { SEM }\end{array}$ & $\begin{array}{l}\text { SUCCE } \\
\text { SSFUL }\end{array}$ & $\begin{array}{c}\text { SUCCESSF } \\
\text { UL }\end{array}$ \\
\hline 10 & $\begin{array}{l}\text { ADONIS } \\
\text { NICOLAS }\end{array}$ & $\begin{array}{c}1 \\
8- \\
1 \\
9 \\
\end{array}$ & $\begin{array}{c}\text { EN1 } \\
02\end{array}$ & $\begin{array}{l}\text { WRITE } \\
\text { ENGLISH }\end{array}$ & $\begin{array}{l}\text { GRADE } \\
12 \mathrm{ABM}\end{array}$ & $\begin{array}{c}180027 \\
82200\end{array}$ & $\begin{array}{c}\text { ELISHA } \\
\text { JAMES } \\
\text { VAQUILAR }\end{array}$ & 98 & $\begin{array}{l}\text { 2ND } \\
\text { SEM }\end{array}$ & $\begin{array}{l}\text { SUCCE } \\
\text { SSFUL }\end{array}$ & $\begin{array}{c}\text { SUCCESSF } \\
\text { UL }\end{array}$ \\
\hline
\end{tabular}


Mary Jane R. Laranang et al., International Journal of Emerging Trends in Engineering Research, 8(9), September 2020, 5445 - 5453

number concerning the student record for grade notification via SMS. The table showed ten successful test trial of sending grade notification of students to the guardians had obtained the desired output.

Table 5: Student grade inquiry test

\begin{tabular}{|c|c|c|c|c|c|}
\hline TRIAL & SID & STUDENTNAME & FP & $\begin{array}{c}\text { EXPECTED } \\
\text { RESULT }\end{array}$ & $\begin{array}{c}\text { ACTUAL } \\
\text { RESULT }\end{array}$ \\
\hline $\mathbf{1}$ & 16001644200 & ANGELIE SILVERIO & BLOB & SUCCESSFUL & SUCCESSFUL \\
\hline $\mathbf{2}$ & 16002497600 & DAISY BRILLANTES & BLOB & SUCCESSFUL & SUCCESSFUL \\
\hline $\mathbf{3}$ & 18002782200 & $\begin{array}{c}\text { ELISHA JAMES } \\
\text { VAQUILAR }\end{array}$ & BLOB & SUCCESSFUL & SUCCESSFUL \\
\hline $\mathbf{4}$ & 16002497601 & LOVE CARE & BLOB & SUCCESSFUL & SUCCESSFUL \\
\hline $\mathbf{5}$ & 16004224300 & REJOICE ULANGCA & BLOB & SUCCESSFUL & SUCCESSFUL \\
\hline $\mathbf{6}$ & 18001547635 & MARY JANE LARANANG & BLOB & SUCCESSFUL & SUCCESSFUL \\
\hline $\mathbf{7}$ & 18001325784 & CARLDWIGHT OCUMEN & BLOB & SUCCESSFUL & SUCCESSFUL \\
\hline $\mathbf{8}$ & 18001325785 & BRYAN L. AGUDONG & BLOB & SUCCESSFUL & SUCCESSFUL \\
\hline $\mathbf{9}$ & 18001325786 & MARK T. ANTONIO & BLOB & SUCCESSFUL & SUCCESSFUL \\
\hline $\mathbf{1 0}$ & 18001325787 & JOHN M BALICAO & BLOB & SUCCESSFUL & SUCCESSFUL \\
\hline
\end{tabular}

Table 6: Importing of student's profile to the system's database using excel file format.

\begin{tabular}{|c|c|c|c|c|c|c|c|}
\hline TRIAL & SID & $\begin{array}{c}\text { STUDENTN } \\
\text { AME }\end{array}$ & $\begin{array}{c}\text { YEAR } \\
\text { LEVEL \& } \\
\text { SECTION }\end{array}$ & $\begin{array}{c}\text { PARENTS } \\
\text { GUARDIAN } \\
\text { S } \\
\end{array}$ & PHONE & $\begin{array}{c}\text { EXPECTED } \\
\text { RESULT }\end{array}$ & $\begin{array}{l}\text { ACTUAL } \\
\text { RESULT }\end{array}$ \\
\hline 1 & 16001644200 & $\begin{array}{l}\text { ANGELIE } \\
\text { SILVERIO }\end{array}$ & $\begin{array}{c}\text { GRADE } 12 \\
\text { ABM }\end{array}$ & $\begin{array}{c}\text { CELSO } \\
\text { APOLTO } \\
\text { SILVERIO }\end{array}$ & $\begin{array}{c}0905172 \\
1234\end{array}$ & SUCCESSFUL & SUCCESSFUL \\
\hline 2 & 16002497600 & $\begin{array}{c}\text { DAISY } \\
\text { BRILLANTE } \\
\text { S }\end{array}$ & $\begin{array}{c}\text { GRADE } 12 \\
\text { ABM }\end{array}$ & $\begin{array}{c}\text { ROMMEL } \\
\text { BRILLANTE } \\
\text { S } \\
\end{array}$ & $\begin{array}{c}0995857 \\
0948\end{array}$ & SUCCESSFUL & SUCCESSFUL \\
\hline 3 & 18002782200 & $\begin{array}{c}\text { ELISHA } \\
\text { JAMES } \\
\text { VAQUILAR }\end{array}$ & $\begin{array}{c}\text { GRADE } 12 \\
\text { ABM }\end{array}$ & $\begin{array}{l}\text { DOLORES } \\
\text { VAQUILAR }\end{array}$ & $\begin{array}{c}0995857 \\
0948\end{array}$ & SUCCESSFUL & SUCCESSFUL \\
\hline 4 & 16002497601 & LOVE CARE & $\begin{array}{c}\text { GRADE } 12 \\
\text { ABM }\end{array}$ & $\begin{array}{c}\text { DAISY } \\
\text { BRILLANTE } \\
\mathrm{S}\end{array}$ & $\begin{array}{c}0939917 \\
0426\end{array}$ & SUCCESSFUL & SUCCESSFUL \\
\hline 5 & 16004224300 & $\begin{array}{c}\text { REJOICE } \\
\text { ULANGCA } \\
\end{array}$ & $\begin{array}{c}\text { GRADE } 12 \\
\text { ABM }\end{array}$ & $\begin{array}{c}\text { ELY } \\
\text { ULANGCA } \\
\end{array}$ & $\begin{array}{c}0966745 \\
9112\end{array}$ & SUCCESSFUL & SUCCESSFUL \\
\hline 6 & 18001547635 & $\begin{array}{l}\text { MARY JANE } \\
\text { LARANANG }\end{array}$ & $\begin{array}{c}\text { GRADE } 12 \\
\text { ABM }\end{array}$ & $\begin{array}{c}\text { SANDY } \\
\text { LARANANG }\end{array}$ & $\begin{array}{c}0935114 \\
5975\end{array}$ & SUCCESSFUL & SUCCESSFUL \\
\hline 7 & 18001325784 & $\begin{array}{c}\text { CARLDWIG } \\
\text { HT } \\
\text { OCUMEN }\end{array}$ & $\begin{array}{c}\text { GRADE } 12 \\
\text { ABM }\end{array}$ & $\begin{array}{l}\text { LYDIA } \\
\text { OCUMEN }\end{array}$ & $\begin{array}{c}0935114 \\
5975\end{array}$ & SUCCESSFUL & SUCCESSFUL \\
\hline 8 & 18001325785 & $\begin{array}{l}\text { BRYAN L. } \\
\text { AGUDONG }\end{array}$ & $\begin{array}{c}\text { GRADE } 12 \\
\text { ABM }\end{array}$ & $\begin{array}{l}\text { MELLISSA } \\
\text { AGUDONG }\end{array}$ & $\begin{array}{c}0935114 \\
5975 \\
\end{array}$ & SUCCESSFUL & SUCCESSFUL \\
\hline 9 & 18001325786 & $\begin{array}{l}\text { MARK T. } \\
\text { ANTONIO }\end{array}$ & $\begin{array}{c}\text { GRADE } 12 \\
\text { ABM }\end{array}$ & $\begin{array}{c}\text { NESTOR } \\
\text { ANTONIO }\end{array}$ & $\begin{array}{c}0935114 \\
5975 \\
\end{array}$ & SUCCESSFUL & SUCCESSFUL \\
\hline 10 & 18001325787 & $\begin{array}{l}\text { JOHN M } \\
\text { BALICAO }\end{array}$ & $\begin{array}{c}\text { GRADE } 12 \\
\text { ABM }\end{array}$ & $\begin{array}{c}\text { MYRNA } \\
\text { BALICAO }\end{array}$ & $\begin{array}{c}0935114 \\
5975 \\
\end{array}$ & SUCCESSFUL & SUCCESSFUL \\
\hline
\end{tabular}




\subsection{ADMINISTRATOR'S BACK UP DATABASE TEST}

For backing up the database to external storage, table 7 shows sample ten instances which $\mathrm{h}$ are successfully backup the system's database by the administrator. The TRIAL column refers to the number of trials, the ID, NAME, and LEVEL columns refer to the profile and account level of the user who tested the backup function of the system. The table showed ten successful test trials of backing up the database had obtained the desired output.

Table 7: Importing of student's profile to the database of the system using excel file

\begin{tabular}{|c|c|c|c|c|c|c|}
\hline TRIAL & ID & NAME & LEVEL & DATECREATED & $\begin{array}{c}\text { EXPECTED } \\
\text { RESULT }\end{array}$ & $\begin{array}{l}\text { ACTUAL } \\
\text { RESULT }\end{array}$ \\
\hline 1 & 1 & RICHMON SANTOS & ADMIN & April 15, 2019 & CREATED & CREATED \\
\hline 2 & 2 & RICHMON SANTOS & ADMIN & April 15, 2019 & CREATED & CREATED \\
\hline 3 & 3 & RICHMON SANTOS & ADMIN & April 15, 2019 & CREATED & CREATED \\
\hline 4 & 4 & RICHMON SANTOS & ADMIN & April 15, 2019 & CREATED & CREATED \\
\hline 5 & 5 & RICHMON SANTOS & ADMIN & April 15, 2019 & CREATED & CREATED \\
\hline 6 & 6 & RICHMON SANTOS & ADMIN & April 15, 2019 & CREATED & CREATED \\
\hline 7 & 7 & RICHMON SANTOS & ADMIN & April 15, 2019 & CREATED & CREATED \\
\hline 8 & 8 & RICHMON SANTOS & ADMIN & April 15, 2019 & CREATED & CREATED \\
\hline 9 & 9 & RICHMON SANTOS & ADMIN & April 15, 2019 & CREATED & CREATED \\
\hline 10 & 10 & RICHMON SANTOS & ADMIN & April 15, 2019 & CREATED & CREATED \\
\hline
\end{tabular}

\subsection{FINAL PROTOTYPE}

Figure 6 shows the prototype result of the established study. In this figure, it includes the system hardware components used. The system software is installed on the server. The display screen serves as an output device for managing and viewing the car park records. The fingerprint scanner is used to capture the fingerprint image of the enrolled student while the thermal printer is used for printing the grades of the student. The broadband stick is used to send an SMS notification to the parents/guardian generated from the system.

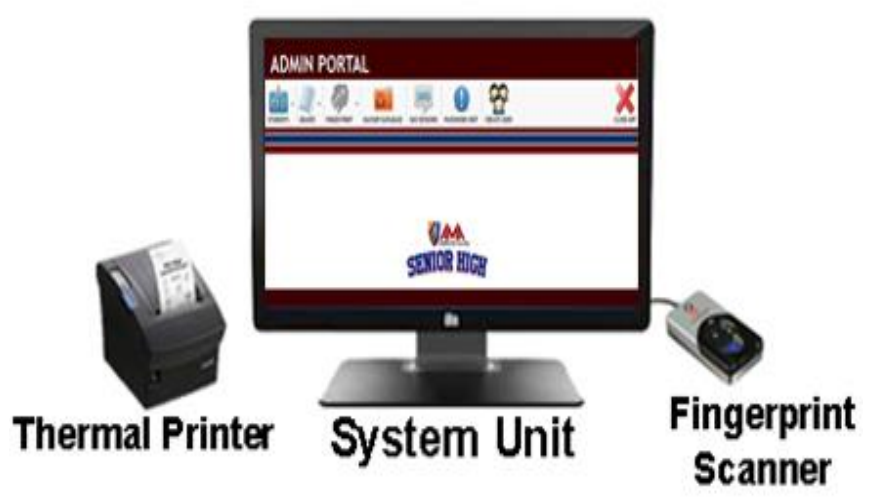

Figure 6: Final prototype

\subsection{SOFTWARE EVALUATION}

An evaluation form was given to the respondents to weigh in the system's functionality, usability, and reliability. The number of respondents was 100 , which composed of random senior high students and faculty of the senior high. The evaluation tool used is the 5-point Likert Scale. Table 8 illustrates the mean perception of the respondents based on the functionality of the system. The results showed that most of the respondents strongly agree on the questions related to its functionality, which is equivalent to an overall weighted average of 4.92 .

On the other hand, table 9 is a tabulated perception of the respondents pertaining to its reliability. The summarized result showed an overall weighted average of 4.87 , which points out that the respondents strongly agree that the system is reliable. Table 10 is the tabulation about the perception of the respondents in terms of the usability of the system. The overall weighted average of 4.64 is the result, which indicates that the respondents describe the usability of the system as "strongly agree." Table 11 is the tabulation about the perception of the respondents in terms of the efficiency of the system. The overall weighted average of 4.88 points out that the respondents strongly agree that the system is efficient.

On the other hand, table 12 is a tabulated perception of the respondents pertaining to its maintainability. The summarized result showed an overall weighted average of 4.90 points out that the respondents strongly agree that the system is maintainable. Lastly, table 13 is the tabulation about the perception of the respondents in terms of the portability of the system. The overall weighted average of 4.95 is its result, which points out that the respondents strongly agree that the system is portable. 
Mary Jane R. Laranang et al., International Journal of Emerging Trends in Engineering Research, 8(9), September 2020, 5445 - 5453

Table 8: Functionality Evaluation Results

\begin{tabular}{|l|l|l|}
\hline Criteria & $\begin{array}{l}\text { Weighted } \\
\text { Average }\end{array}$ & Description \\
\hline $\begin{array}{l}\text { Functions required for the } \\
\text { system is implemented } \\
\text { (suitability) }\end{array}$ & 4.90 & $\begin{array}{l}\text { Strongly } \\
\text { Agree }\end{array}$ \\
\hline $\begin{array}{l}\text { Functions provide correct } \\
\text { information (accurateness) }\end{array}$ & 4.90 & $\begin{array}{l}\text { Strongly } \\
\text { Agree }\end{array}$ \\
\hline $\begin{array}{l}\text { Ease of connecting with other } \\
\text { systems is provided } \\
\text { (interoperability) }\end{array}$ & 4.90 & $\begin{array}{l}\text { Strongly } \\
\text { Agree }\end{array}$ \\
\hline $\begin{array}{l}\text { Functions meet specifications } \\
\text { (Compliance) }\end{array}$ & 4.90 & $\begin{array}{l}\text { Strongly } \\
\text { Agree }\end{array}$ \\
\hline $\begin{array}{l}\text { Substantial security is } \\
\text { provided(Security) }\end{array}$ & $\mathbf{5 . 0 0}$ & $\begin{array}{l}\text { Strongly } \\
\text { Agree }\end{array}$ \\
\hline Overall Weighted Average & $\mathbf{4 . 9 2}$ & $\begin{array}{l}\text { Strongly } \\
\text { Agree }\end{array}$ \\
\hline
\end{tabular}

Table 9: Reliability Evaluation Results

\begin{tabular}{|l|l|l|}
\hline Criteria & $\begin{array}{l}\text { Weighted } \\
\text { Average }\end{array}$ & Description \\
\hline $\begin{array}{l}\text { The software includes no } \\
\text { errors(maturity) }\end{array}$ & 4.85 & $\begin{array}{l}\text { Strongly } \\
\text { Agree }\end{array}$ \\
\hline $\begin{array}{l}\text { The system continues to } \\
\text { operate at the slightest } \\
\text { disturbance (fault tolerance) }\end{array}$ & 4.80 & $\begin{array}{l}\text { Strongly } \\
\text { Agree }\end{array}$ \\
\hline $\begin{array}{l}\text { System operations are restored } \\
\text { quickly when a failure } \\
\text { occurs(recoverability) }\end{array}$ & 4.95 & $\begin{array}{l}\text { Strongly } \\
\text { Agree }\end{array}$ \\
\hline Overall Weighted Average & $\mathbf{4 . 8 7}$ & $\begin{array}{l}\text { Strongly } \\
\text { Agree }\end{array}$ \\
\hline
\end{tabular}

Table 10: Usability Evaluation Results

\begin{tabular}{|l|l|l|}
\hline Criteria & $\begin{array}{l}\text { Weighted } \\
\text { Average }\end{array}$ & Description \\
\hline $\begin{array}{l}\text { Easy to operate or } \\
\text { navigate(understandability) }\end{array}$ & 5.00 & $\begin{array}{l}\text { Strongly } \\
\text { Agree }\end{array}$ \\
\hline $\begin{array}{l}\text { Easy to remember system } \\
\text { procedure(learnability) }\end{array}$ & 4.95 & $\begin{array}{l}\text { Strongly } \\
\text { Agree }\end{array}$ \\
\hline $\begin{array}{l}\text { Allow easy operation } \\
\text { management(operability) }\end{array}$ & 4.95 & $\begin{array}{l}\text { Strongly } \\
\text { Agree }\end{array}$ \\
\hline Overall Weighted Average & $\mathbf{4 . 9 7}$ & $\begin{array}{l}\text { Strongly } \\
\text { Agree }\end{array}$ \\
\hline
\end{tabular}

Table 11: Efficiency Evaluation Results

\begin{tabular}{|l|l|l|}
\hline Criteria & $\begin{array}{l}\text { Weighted } \\
\text { Average }\end{array}$ & Description \\
\hline $\begin{array}{l}\text { Provides good responses with } \\
\text { minimal time (time behavior) }\end{array}$ & 4.85 & $\begin{array}{l}\text { Strongly } \\
\text { Agree }\end{array}$ \\
\hline $\begin{array}{l}\text { Allows effective use of system } \\
\text { resources(resource behavior) }\end{array}$ & 4.90 & $\begin{array}{l}\text { Strongly } \\
\text { Agree }\end{array}$ \\
\hline Overall Weighted Average & $\mathbf{4 . 8 8}$ & $\begin{array}{l}\text { Strongly } \\
\text { Agree }\end{array}$ \\
\hline
\end{tabular}

Table 12: Maintainability Evaluation Results

\begin{tabular}{|l|l|l|}
\hline Criteria & $\begin{array}{l}\text { Weighted } \\
\text { Average }\end{array}$ & Description \\
\hline $\begin{array}{l}\text { Allows easy analysis of design } \\
\text { documents and programs when } \\
\text { an error is found(analyzability) }\end{array}$ & 4.90 & $\begin{array}{l}\text { Strongly } \\
\text { Agree }\end{array}$ \\
\hline $\begin{array}{l}\text { Allows easy modification of } \\
\text { the system(changeability) }\end{array}$ & 4.90 & $\begin{array}{l}\text { Strongly } \\
\text { Agree }\end{array}$ \\
\hline $\begin{array}{l}\text { The modification does not } \\
\text { affect the whole system } \\
\text { (stability) }\end{array}$ & 4.85 & $\begin{array}{l}\text { Strongly } \\
\text { Agree }\end{array}$ \\
\hline $\begin{array}{l}\text { Strenuous tests are not required } \\
\text { after modification is } \\
\text { made(testability) }\end{array}$ & 4.95 & $\begin{array}{l}\text { Strongly } \\
\text { Agree }\end{array}$ \\
\hline Overall Weighted Average & $\mathbf{4 . 9 0}$ & $\begin{array}{l}\text { Strongly } \\
\text { Agree }\end{array}$ \\
\hline
\end{tabular}

Table 13: Portability Evaluation Results

\begin{tabular}{|l|l|l|}
\hline Criteria & $\begin{array}{l}\text { Weighted } \\
\text { Average }\end{array}$ & Description \\
\hline $\begin{array}{l}\text { Accessible in a different } \\
\text { environment(adaptability) }\end{array}$ & 5.00 & $\begin{array}{l}\text { Strongly } \\
\text { Agree }\end{array}$ \\
\hline $\begin{array}{l}\text { Provide easy } \\
\text { installation(installability) }\end{array}$ & 4.95 & $\begin{array}{l}\text { Strongly } \\
\text { Agree }\end{array}$ \\
\hline $\begin{array}{l}\text { Meets design } \\
\text { specifications(conformance) }\end{array}$ & 4.85 & $\begin{array}{l}\text { Strongly } \\
\text { Agree }\end{array}$ \\
\hline $\begin{array}{l}\text { Allows replacement easily with } \\
\text { other software(replaceability) }\end{array}$ & 5.00 & $\begin{array}{l}\text { Strongly } \\
\text { Agree }\end{array}$ \\
\hline Overall Weighted Average & $\mathbf{4 . 9 5}$ & $\begin{array}{l}\text { Strongly } \\
\text { Agree }\end{array}$ \\
\hline
\end{tabular}

\section{CONCLUSION}

The "Students' Grade Inquiry Kiosk Using Biometric Fingerprint Authentication With SMS Notification To Parents" is a system primarily to view the grades of the students. The main innovation of the system is the use of fingerprint technology for more efficient and reliable monitoring, recording, and viewing of grades results of the students.

Based on the tasks engaged in the establishment of the system, which includes problem identification, formulation of objectives, development, tests, and evaluations conducted, it was proven that the system performs its intended functions. The established system is capable of importing students' information and grades. It can access grades with fingerprint authentication as well as SMS notification to parents/guardians. Finally, it is established that the system software is easy to use and understand, presents accurate and complete data, and is very useful to the grade inquiry of the school. 


\section{REFERENCES}

[1] M. Shirali-Shahreza, "Design and implementation of three different methods for announcing exam grades on the web," Proceedings - The 8th IEEE International Conference on Advanced Learning Technologies, ICALT 2008, pp. 335-337, 2008.

[2] M. Ridzuan and B. Hashim, "Combination of Fingerprint and Hidden Load Cell ( CFH )," Journal of Computing Technologies and Creative Content, vol. 2, no. 1, pp. 63-72, 2017.

[3] Rofiq, "Fingerprint Technology," https://decdeg.com/fingerprint-technology/, 2020. .

[4] M. D. Rahmatya and M. F. Wicaksono, "Design of Student Attendance Information System with Fingerprints," IOP Conference Series: Materials Science and Engineering, pp. 1-5, 2019.

[5] E. B. Panganiban, "Microcontroller-based Wearable Blood Pressure Monitoring Device with GPS and SMS Feature through Mobile App," International Journal of Emerging Trends in Engineering Research, vol. 7, no. 6, pp. 32-35, 2019.

[6] E. B. Panganiban, "Automated hazardous gas detecting robot using wireless sensor networks with GSM-SMS alert and fire control system for households," International Journal of Advanced Trends in Computer Science and Engineering, vol. 8, no. 3, pp. 804-809, 2019.

[7] I. T. Plata, E. B. Panganiban, B. B. Bartolome, F. E. R. Labuanan, and A. C. Taracatac, "A Concept of Cassava Phytoplasma Disease Monitoring and Mapping System using GIS and SMS Technology," International Journal of Advanced Trends in Computer Science and Engineering, vol. 8, no. 6, pp. 3357-3361, 2019.

[8] S. So, "The Development of a SMS-based Teaching and Learning System," Journal of Educational Technology Development and Exchange, vol. 2, no. 1, pp. 113-124, 2009.

[9] R. G. Lumauag, "SENT SMS : School Event Notification Through SMS," Asia Pacific Journal of Multidisciplinary Research, vol. 4, no. 4, pp. 61-68, 2016.

[10] M. S. Shahreza, "M-quiz by SMS," Proceedings Sixth International Conference on Advanced Learning Technologies, ICALT 2006, pp. 726-729, 2006.

[11] M. Shirali-Shahreza, "Taking multiple-choise quiz by SMS on mobile phones including analyzing property," Journal of Computers (Finland), vol. 2, no. 2, pp. 53-60, 2007.

[12] I. N. Mohamed, A. T. Siddiqui, S. Ajaz, and S. Mohamed Idhris, "Student Information Report System with SMS (SIRS)," 2016 International Conference on Computer Communication and Informatics, ICCCI 2016, pp. 7-10, 2016.

[13] K. Jaikumar, M. Santosh Kumar, S. Rajkumar, and A. Sakthivel, "Fingerprint Based Student Attendance
System With Sms Alert To Parents," International Journal of Research in Engineering and Technology, vol. 04, no. 02, pp. 293-297, 2015.

[14] Z. Alansari, "SMS-Enabled Student Information System," 2011.

[15] I. R. Murmanto and D. F. Murad, "Computerizing Values and Accessing Values via SMS," ULTIMA InfoSys, vol. X, no. 2, pp. 76-81, 2019.

[16] L. Agustin, F. Flores, S. Galindo, and R. Casabar, "Utilizing Sms And Student Portals : Academic Grades Viewing And Notification," Journal of Global Research in Computer Science, vol. 9, no. 12, pp. 1-5, 2018.

[17] R.R. Codilan, "Short Messaging Service (SMS) Based Grade Inquiry System," EPRA Internation Journal of Research \& Development, vol. 4, no. 6, pp. 20-27, 2019.

[18] E. B. Panganiban and J. P. Bermusa, "Simplified Barcode-Based Point Of Sales And Inventory Management System With Replenishment Decision," International Journal of Scientific \& Technology Research, vol. 9, no. 03, pp. 597-600, 2020. 